
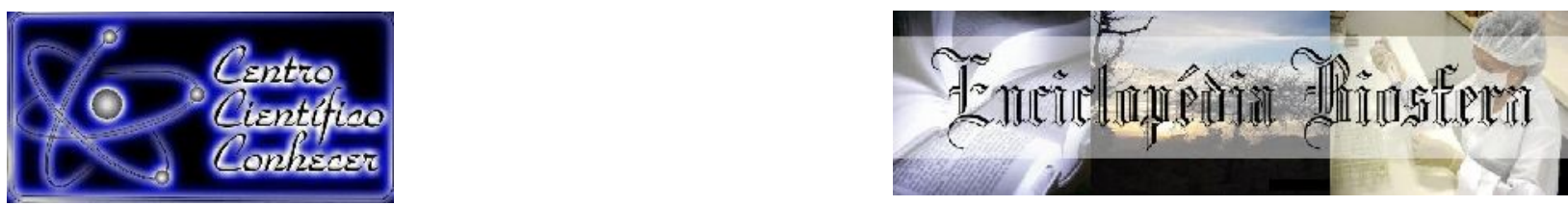

\title{
ESTRATÉGIA MULTIMODAL: PROPOSTA EDUCACIONAL VOLTADA À SEGURANÇA DO PACIENTE
}

\section{Ednólia Gomes Varjão Fernandes ${ }^{1}$, Isabela Gomes dos Santos ${ }^{2}$, Raphaela Silva ${ }^{3}$, Adriana Santos Prado Sadoyama ${ }^{4}$, Geraldo Sadoyama ${ }^{5}$}

${ }^{1}$ Enfermeira, Especialista em Enfermagem do Trabalho, Unidade de Terapia Intensiva e Docência do Ensino Superior, Mestranda no Programa de Pós-Graduação em Gestão Organizacional pela Universidade Federal de Goiás - Regional Catalão-GO, Brasil. E-mail ednoliavihart@gmail.com

${ }^{2}$ Graduada em Ciências Biológicas, Mestranda no Programa de Pós-Graduação em Gestão Organizacional pela Universidade Federal de Goiás - Regional Catalão-GO, Brasil.

${ }^{3}$ Nutricionista, Especialista em Nutrição Clínica e Nutrição Oncológica, Mestranda no Programa de Pós-Graduação em Gestão Organizacional pela Universidade Federal de Goiás - Regional Catalão-GO, Brasil.

${ }^{4}$ Doutora em Linguística e Língua Portuguesa, Professora do Programa de Pós-Graduação em Gestão Organizacional pela Universidade Federal de Goiás - Regional Catalão-GO, Brasil.

${ }^{5}$ Doutor em Imunologia e Parasitologia, Professor do Programa de Pós-Graduação em Gestão Organizacional pela Universidade Federal de Goiás - Regional Catalão-GO, Brasil.

\section{Recebido em: 04/10/2019 - Aprovado em: 30/11/2019 - Publicado em: 15/12/2019} DOI: 10.18677/EnciBio 2019B27

\section{RESUMO}

Estudiosos da área de gestão em saúde têm se preocupado em desenvolver estratégias educativas e inovadoras que promovam a segurança do paciente e a qualidade da assistência à saúde. Diante do exposto, o objetivo deste estudo foi sistematizar o conhecimento aplicado à estratégia multimodal em segurança do paciente. Esta pesquisa tratou-se de uma revisão integrativa que buscou sintetizar e discutir as principais ações, recomendações e lacunas acerca da temática, utilizando as bases de dados SCIELO e BVS, incluindo artigos com abordagem qualitativa que responderam à questão norteadora e aos objetivos propostos, publicados em periódicos científicos, disponíveis na íntegra e de forma gratuita, publicados no idioma português, inglês, e espanhol, durante período de 2014 a 2019, totalizando quatro artigos que compuseram a amostra do estudo. A estratégia multimodal tratase de uma proposta educacional que pode ser aplicada em diferentes contextos com o objetivo de estimular melhorias organizacionais. Contudo, a Organização Mundial da Saúde publicou um manual direcionado às instituições de saúde para a implantação da estratégia multimodal com o intuito de melhorar a prática de higienização das mãos. Por esse motivo, todos os artigos analisados nesta revisão integrativa referem-se à avaliação dessa estratégia multimodal relacionada à higienização das mãos. Conclui-se que é imprescindível garantir a implantação, continuidade e manutenção das iniciativas de segurança do paciente, assim como no monitoramento e divulgação dos resultados associados às iniciativas de melhorias contínuas. Garantir segurança do paciente é fruto do esforço e comprometimento de toda a equipe envolvida direta ou indiretamente na assistência ao paciente.

PALAVRAS-CHAVE: Estratégia Multimodal, Higienização de Mãos, Segurança do Paciente. 


\title{
MULTIMODAL STRATEGY: EDUCATIONAL PROPOSAL FOR PATIENT SAFETY
}

\begin{abstract}
Health management scholars have been concerned with developing innovative and educational strategies that promote patient safety and the quality of health care. Given the above, the objective of this study was to systematize the knowledge applied to the multimodal strategy in patient safety. This research is an integrative review that sought to synthesize and discuss the main actions, recommendations and gaps about the theme, using the SciELO and BVS databases, including articles with qualitative approach that answered the guiding question and the proposed objectives, published. in scientific journals, available in their entirety and free of charge, published in Portuguese, English, and Spanish, from 2014 to 2019, totaling 4 articles that comprised the study sample. The multimodal strategy is an educational proposal that can be applied in different contexts in order to stimulate organizational improvements. However, the World Health Organization has published a manual directed to health institutions for the implementation of the multimodal strategy in order to improve the practice of hand hygiene. For this reason, all articles analyzed in this integrative review refer to the evaluation of this multimodal strategy related to hand hygiene. It is concluded that it is essential to ensure the implementation, continuity and maintenance of patient safety initiatives, as well as the monitoring and dissemination of results associated with continuous improvement initiatives. Ensuring patient safety is the result of the effort and commitment of all staff directly or indirectly involved in patient care.
\end{abstract}

KEYWORDS: Multimodal Strategy, Hand Hygiene, Patient Safety.

\section{INTRODUÇÃO}

Os avanços científicos e tecnológicos, assim como a ampliação de acesso aos serviços de saúde contribuíram para que os índices de morbidade e mortalidade decrescessem nas últimas décadas. Contudo, há relatos de eventos adversos, isto é, incidentes, que podem causar danos irreversíveis à saúde dos pacientes e outros prejuízos de ordem social e econômica (REIS et al., 2013). Para evitar que eventos adversos aconteçam é necessário que a instituição de saúde zele pela segurança do paciente e, para tanto, desenvolva ações que estimulem o desenvolvimento de uma cultura organizacional comprometida com o cuidado (ANDRADE et al., 2018).

Compreende-se cultura organizacional como um conjunto de competências técnicas, práticas assistenciais, hábitos, atitudes, crenças e valores que são intrínsecos à equipe multiprofissional de uma organização. Já a cultura de segurança do paciente orienta como os profissionais devem agir e se comportar para garantir que eventos adversos não aconteçam. Por isso, pode-se dizer que uma cultura de segurança revela o comprometimento da instituição com o bem-estar de seus pacientes e com a qualidade dos serviços prestados (SILVA et al., 2018).

A cultura de segurança do paciente é influenciada de forma positiva por campanhas educativas e pela qualificação contínua dos profissionais de saúde. Deve-se isso ao fato das atividades relacionadas à educação em saúde permitirem que novas tecnologias, práticas e novos conhecimentos técnico-científicos sejam incorporados ao sistema. Assim, pode-se dizer que políticas institucionais que estimulam a educação permanente dos profissionais de saúde são fundamentais para promover mudanças organizacionais (WEGNER et al., 2016). 
Por esse motivo, a Organização Mundial da Saúde (OMS) desenvolve várias atividades educativas com o objetivo de promover atendimentos seguros e aumentar a qualidade dos serviços de saúde prestados à população. Dentre as várias iniciativas conduzidas pela OMS, destacam-se os guias para a implantação de estratégia multimodal que previnem a ocorrência de eventos adversos. Essas estratégias são ferramentas que instruem como as unidades de saúde devem planejar, avaliar as condições prévias, implantar um novo processo, acompanhar os resultados e desenvolver um plano de ação (OMS, 2008).

Pode-se dizer que o relatório "To Error is Human: Building a safer health system" (Errar é Humano: Construindo um sistema de saúde mais seguro), publicado em 2000, despertou a atenção dos profissionais de saúde para a importância da segurança do paciente. Esse relatório revelou que a assistência médica nos Estados Unidos não é tão segura, pois milhares de pessoas morrem todos os anos devido a eventos adversos decorrentes de erros (KOHN et al., 2000). Em países menos avançados, como o Brasil, estima-se que a situação seja ainda pior (JHA et al., 2013).

Riscos a eventos adversos existem em todos os lugares onde há assistência à saúde (MELLO; BARBOSA, 2013). Os principais erros estão relacionados à identificação do paciente, medicação, acidentes e infecção (DUARTE et al., 2016). Ademais, fatores ambientais, como a desordem, assim como as más condições psicológicas ou fisiológicas do profissional de saúde podem contribuir para a ocorrência de erros. Mangilli e colaboradores (2017) também apontam que a falta de atenção, conhecimento e a sobrecarga de trabalho corroboram para a ocorrência de falhas na assistência.

Algumas falhas na assistência, contudo, não são provenientes de erros e, portanto, não podem ser evitadas. Porém, a maioria dos eventos adversos ocorre devido a erros no planejamento ou na execução de uma tarefa. Logo, esses incidentes são considerados como evitáveis e constituem um problema de qualidade na assistência. Por isso, diversos órgãos, como a OMS, desenvolvem estratégias para detectar e prevenir a incidência de eventos adversos que causam tantos prejuízos ao sistema de saúde (LANZILLOTTI et al., 2015).

Para que a ocorrência de eventos adversos seja atenuada, é necessário que os profissionais de saúde, inicialmente, reconheçam o problema e compreendam quais são as causas e consequências dos incidentes. Muitos profissionais, no entanto, não sabem lidar com esses incidentes e sentem-se frustrados. Além disso, muitos temem por sanções disciplinares e omitem suas falhas. Isso significa dizer que essa temática precisa ser problematizada sob uma nova perspectiva para que as falhas sejam reconhecidas como oportunidades para aprender e aprimorar a assistência. Portanto, médicos, enfermeiros, técnicos e gestores devem entender que o cuidar é uma ação humana suscetível a falhas (WEGNER et al., 2016).

Estudos também indicam que a maioria dos incidentes ocorre devido a falhas no sistema organizacional e não devido à falta de competência de um único profissional. A partir desse contexto, o Programa Nacional de Segurança do Paciente (PNSP), estabelecido pelo Ministério da Saúde em 2013, propôs o desenvolvimento de uma cultura de segurança que encoraje os profissionais de saúde a notificarem a incidência de eventos adversos. Espera-se, dessa maneira, promover o aprendizado coletivo e melhorias organizacionais. Assim, desde julho de 2014, tornou-se obrigatório que os profissionais de saúde registrem a ocorrência de eventos adversos no Sistema de Notificações para a Vigilância Sanitária (Notivisa) (MAIA et al., 2018). 
Além disso, para promover um ambiente mais seguro aos pacientes, os hospitais e clínicas devem promover treinamentos contínuos, utilizar protocolos atualizados e constituir comitês de segurança. Isso significa dizer que os gestores e a equipe multiprofissional de saúde devem explorar as causas dos incidentes e elaborar estratégias que previnem a ocorrência de erros e eventos adversos. Podese dizer então que medidas educativas contribuem para atenuar os problemas relacionados à falta de segurança (TOBIAS et al., 2016).

Por esse motivo, a Organização Mundial de Saúde e a Agência Nacional de Vigilância Sanitária (ANVISA) desenvolvem vários instrumentos educativos e inovadores com o objetivo de reduzir os riscos nos serviços de atendimento e, como consequência, aprimorar a segurança e a qualidade das instituições de saúde. $\mathrm{O}$ guia para a implantação da estratégia multimodal para a melhoria da higienização das mãos, por exemplo, tem como propósito reduzir o número de eventos adversos relacionados às infecções que agravam as taxas de morbidade e mortalidade em todo o país (ANVISA, 2008).

Estudiosos da área de gestão em saúde também têm se preocupado em desenvolver estratégias educativas e inovadoras que promovam a segurança do paciente e a qualidade da assistência à saúde. Ademais, a preocupação com o paciente tornou-se um fator competitivo no cenário mercadológico atual. Assim, diante do exposto, este estudo teve como objetivo sistematizar o conhecimento aplicado à estratégia multimodal em segurança do paciente. Esta pesquisa tratou-se de uma revisão integrativa que buscou sintetizar e discutir as principais ações, recomendações e lacunas acerca da temática.

\section{MATERIAL E MÉTODOS}

Esta pesquisa buscou responder a seguinte questão norteadora: A estratégia multimodal tem aplicabilidade na capacitação e atualização voltada à segurança do paciente? Para a realização desta pesquisa, optou-se por utilizar o método de revisão integrativa $(\mathrm{RI})$, o qual é baseado em evidências e apresenta como proposta de desenvolvimento a realização de uma análise do conhecimento existente sobre o tema, com buscas em pesquisas já realizadas (MENDES et al., 2008).

O nível e qualidade de evidência foram aplicados no material que se adequaram aos critérios de inclusão. Foi utilizado seis níveis de evidência para avaliar os artigos: Nível I - Metanálise de múltiplos estudos controlados; nível II Estudo experimental individual; nível III - estudo quase experimental como grupo único, não randomizado, controlado, com pré e pós-teste, ou estudos tipo caso controle; nível IV - estudo não experimental como pesquisa descritiva correlacional, pesquisa qualitativa ou estudo de caso; nível V - relatórios de casos ou dados obtidos sistematicamente, de qualidade verificável ou dados de programação ou avaliação; nível VI - opinião de autoridades respeitadas (como autores conhecidos nacionalmente) baseados em sua experiência clínica ou a opinião de um comitê de peritos incluindo suas interpretações de informações não baseada em pesquisa. Este nível também inclui a opinião de órgãos de regulamentação ou legais

Para a estratégia de identificação e seleção dos estudos, foram buscadas publicações indexadas nas bases de dados Scientific Eletronic Library Online (Scielo) e Biblioteca Virtual em Saúde - Portal Regional BVS. Nas referidas bases foram inseridos os descritores "Estratégia Multimodal" e "Segurança do Paciente". Adotaram-se os seguintes critérios de inclusão: artigos completos publicados no período de 2014 a 2019, disponíveis eletronicamente nos idiomas português, espanhol ou inglês e que abordassem o conhecimento dentro da temática proposta. Excluíram-se os artigos duplicados, artigos de 
revisão integrativa e artigos que não fossem pertinentes ao objetivo desta pesquisa. A Figura 1 representa o mecanismo de busca utilizado pelos autores deste trabalho.
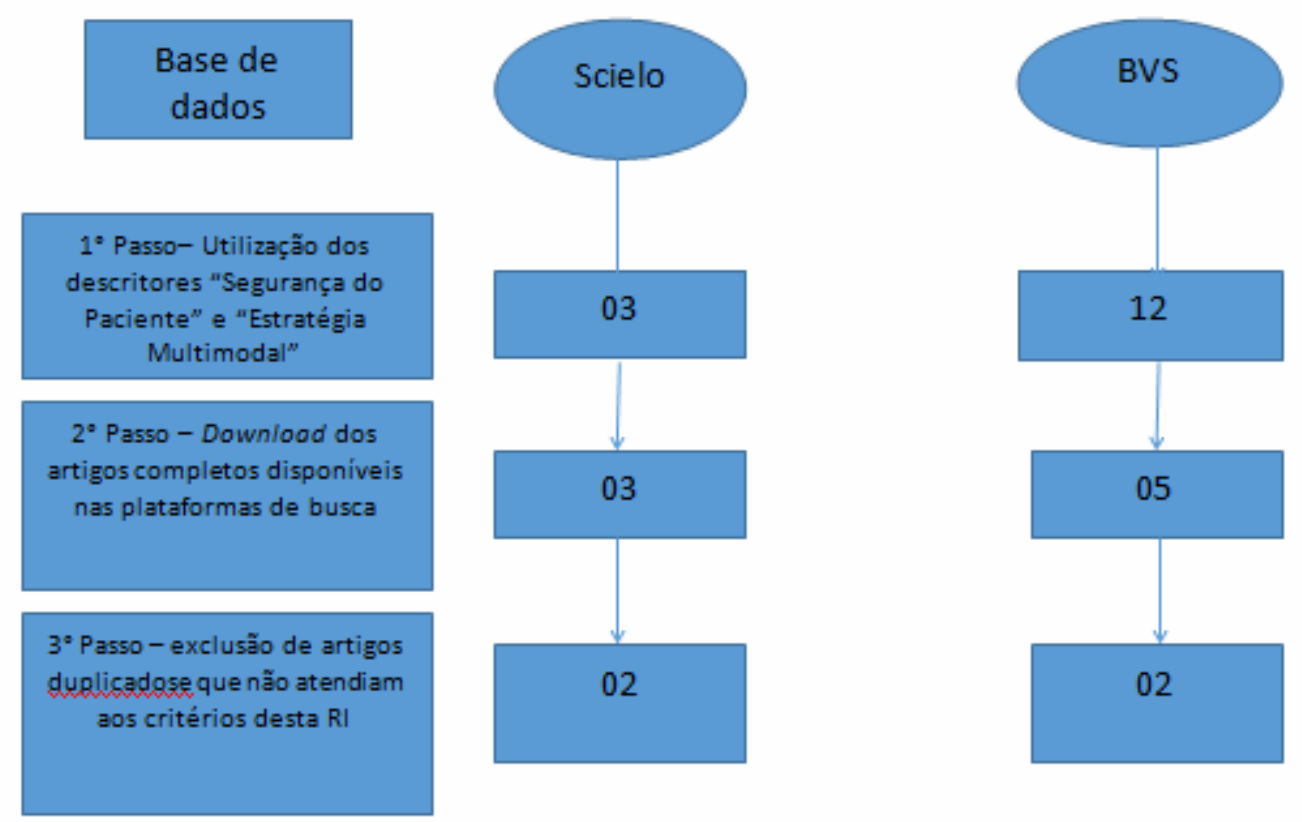

FIGURA 1- Fluxograma do processo de identificação, seleção, elegibilidade e inclusão dos estudos.

Fonte: Elaborado pelos autores. Catalão, GO, Brasil, (2019).

\section{RESULTADOS E DISCUSSÃO}

Ao total, apenas quatro artigos foram considerados como adequados para esta revisão. As principais informações dos artigos selecionados estão descritas no Quadro 1.

\begin{tabular}{|c|c|c|c|c|}
\hline Autores & Título & $\begin{array}{c}\text { Idioma / } \\
\text { Base de } \\
\text { dados/ Ano } \\
\text { de } \\
\text { publicação }\end{array}$ & $\begin{array}{l}\text { Delineament } \\
\text { o do estudo/ } \\
\text { Nível de } \\
\text { evidência }\end{array}$ & Principais resultados/Conclusão \\
\hline $\begin{array}{l}\text { Magnag } \\
\text { o et al. }\end{array}$ & $\begin{array}{l}\text { Infraestr } \\
\text { utura } \\
\text { para } \\
\text { higieniz } \\
\text { ação } \\
\text { das } \\
\text { mãos } \\
\text { em um } \\
\text { hospital } \\
\text { universit } \\
\text { ário }\end{array}$ & $\begin{array}{l}\text { Português/ } \\
\text { Scielo/ } 2019\end{array}$ & $\begin{array}{l}\text { Estudo } \\
\text { observaciona } \\
\text { I com } \\
\text { delineamento } \\
\text { transversal } \\
\text { descritivo. de } \\
\text { Nível de } \\
\text { evidência IV. }\end{array}$ & $\begin{array}{l}\text { A adesão à prática de higienização das mãos (HM) está } \\
\text { associada à infraestrutura do ambiente hospitalar. A } \\
\text { ausência de pias adequadas, dispensadores de } \\
\text { sabonete líquido e álcool gel, toalhas descartáveis e } \\
\text { lixeiras pode inibir essa prática. Assim, a partir desse } \\
\text { pressuposto, o estudo avaliou as condições físicas de } \\
\text { um hospital universitário. Foram então analisadas } 18 \\
\text { unidades de internação e } 16 \text { coordenadores foram } \\
\text { entrevistados. Todos os coordenadores afirmaram que } \\
\text { as unidades possuem soluções alcoólicas para a HM. } \\
\text { Entretanto, observou-se que apenas a UTI adulto } \\
\text { possuía frascos de álcool próximos aos leitos. Observou- } \\
\text { se também que poucos profissionais de saúde utilizam } \\
\text { frascos de bolso e que muitas pias possuem fechamento } \\
\text { manual.Os coordenadores enfermeiros, por outro lado, } \\
\text { afirmaram que os profissionais de saúde receberam } \\
\text { capacitação sobre os protocolos e observou-se que ao } \\
\text { lado dos dispensadores localizados nos corredores há } \\
\text { cartazes que orientam como os profissionais de saúde, } \\
\text { pacientes e acompanhantes devem realizar a } \\
\text { higienização das mãos. Concluiu-se, portanto, que a }\end{array}$ \\
\hline
\end{tabular}




\begin{tabular}{|c|c|c|c|c|}
\hline & & & & $\begin{array}{l}\text { infraestrutura do local de pesquisa está em parte de } \\
\text { acordo com a resolução RDC no } 50 \text { que dispõe sobre a } \\
\text { estrutura física ideal dos estabelecimentos assistenciais } \\
\text { de saúde. }\end{array}$ \\
\hline $\begin{array}{l}\text { Vasconc } \\
\text { elos et } \\
\text { al. }\end{array}$ & $\begin{array}{l}\text { Adesão } \\
\text { à } \\
\text { higieniz } \\
\text { ação } \\
\text { das } \\
\text { mãos } \\
\text { pela } \\
\text { equipe } \\
\text { de } \\
\text { enferma } \\
\text { gem em } \\
\text { unidade } \\
\text { de } \\
\text { terapia } \\
\text { intensiv } \\
\text { a }\end{array}$ & $\begin{array}{l}\text { Português/ } \\
\text { Scielo/2018 }\end{array}$ & $\begin{array}{l}\text { Pesquisa } \\
\text { observaciona } \\
\text { I com } \\
\text { delineamento } \\
\text { transversal } \\
\text { descritivo. } \\
\text { Nível de } \\
\text { evidência IV. }\end{array}$ & $\begin{array}{l}\text { A Organização Mundial da Saúde orienta que os } \\
\text { profissionais de saúde higienizem as mãos em cinco } \\
\text { momentos: antes de estabelecer contato com o paciente, } \\
\text { antes de realizar algum procedimento asséptico, após } \\
\text { algum risco decorrente da exposição a fluídos corporais, } \\
\text { após estabelecer contato com o paciente e após contatar } \\
\text { áreas próximas ao paciente. Esses cuidados previnem } \\
\text { infecções e aumentam a segurança dos pacientes e } \\
\text { profissionais de saúde. A partir desse contexto, os } \\
\text { autores avaliaram se os enfermeiros e técnicos de } \\
\text { enfermagem que trabalham em uma Unidade de Terapia } \\
\text { Intensiva cumprem ou não as recomendações da OMS } \\
\text { no que tange à higienização das mãos. Os } \\
\text { pesquisadores utilizaram o Instrumento Adaptado do } \\
\text { Manual para Observadores - Estratégia Multimodal da } \\
\text { OMS para melhoria da HM. Observou-se que a } \\
\text { higienização das mãos ocorre geralmente após o contato } \\
\text { com os pacientes. Observou-se também que os } \\
\text { profissionais não higienizam as mãos antes de realizar } \\
\text { algum procedimento asséptico. Muitos profissionais não } \\
\text { receberam treinamentos nos últimos dois anos sobre a } \\
\text { estratégia multimodal que orienta os profissionais a } \\
\text { terem boas práticas de higiene. Os dados mostraram } \\
\text { que não há diferença estatística entre técnicos e } \\
\text { enfermeiros quando se trata de higienização das mãos. } \\
\text { Os pesquisadores concluíram que a equipe de } \\
\text { enfermagem apresenta baixa adesão às orientações da } \\
\text { OMS quando se trata de higienização das mãos, } \\
\text { possivelmente, devido à falta a de } \\
\text { treinamento/capacitação e ausência de infraestrutura } \\
\text { adequada. }\end{array}$ \\
\hline $\begin{array}{l}\text { Ferreira, } \\
\text { S.A. }\end{array}$ & $\begin{array}{l}\text { Implanta } \\
\text { ção do } \\
\text { Projeto: } \\
\text { Seguran } \\
\text { ça do } \\
\text { Pacient } \\
\text { e "Mãos } \\
\text { limpas } \\
\text { são } \\
\text { mãos } \\
\text { mais } \\
\text { seguras } \\
\text { ". Áaliaçã } \\
\text { o da } \\
\text { etapa } \\
2011- \\
2012 . \\
\text { Estado } \\
\text { de São } \\
\text { Paulo. }\end{array}$ & $\begin{array}{l}\text { Português/B } \\
\text { VS/2014. }\end{array}$ & $\begin{array}{l}\text { Estudo } \\
\text { transversal e } \\
\text { descritivo. } \\
\text { Nível de } \\
\text { evidência IV. }\end{array}$ & $\begin{array}{l}\text { A baixa adesão à prática de higienização das mãos é um } \\
\text { problema em todo o mundo. Por isso, a OMS } \\
\text { desenvolveu um guia para a melhoria dessa prática. } \\
\text { Esse guia contempla mudanças estruturais, atividades } \\
\text { de capacitação, avaliação do processo, utilização de } \\
\text { lembretes e desenvolvimento de um clima de segurança } \\
\text { institucional. A partir desse contexto, o Centro de } \\
\text { Vigilância Epidemiológica desenvolveu o projeto "Mãos } \\
\text { limpas são mãos mais seguras" com o intuito de aplicar } \\
\text { essas estratégias nos hospitais públicos, privados e } \\
\text { filantrópicos do estado de São Paulo. Esse estudo, } \\
\text { portanto, teve como objetivo avaliar a eficácia dessas } \\
\text { estratégias. O projeto supracitado desenvolveu-se em } \\
\text { quatro etapas, a saber: preparação da unidade, } \\
\text { aplicação dos instrumentos de avaliação, capacitação } \\
\text { dos profissionais de saúde e avaliação final. Os } \\
\text { indicadores de melhoria utilizados antes e após a } \\
\text { intervenção educativa foram: mensuração do consumo } \\
\text { de produto alcoólico, análise da estrutura da unidade e } \\
\text { aplicação de questionários com o intuito de verificar a } \\
\text { percepção e os conhecimentos dos profissionais de } \\
\text { saúde sobre a prática de higienização das mãos. } \\
\text { Constatou-se que o projeto contribuiu para que os } \\
\text { hospitais desenvolvessem ações para aumentar a }\end{array}$ \\
\hline
\end{tabular}




\begin{tabular}{|c|c|c|c|c|}
\hline & & & & $\begin{array}{l}\text { adesão à prática de higienização das mãos. Contudo, a } \\
\text { pesquisadora afirma que essas ações precisam ser } \\
\text { sustentadas e expandidas. }\end{array}$ \\
\hline $\begin{array}{l}\text { Fariñas- } \\
\text { Alvarez, } \\
\text { et al. }\end{array}$ & $\begin{array}{l}\text { Estrateg } \\
\text { ia } \\
\text { multimo } \\
\text { dal para } \\
\text { lamejora } \\
\text { de } \\
\text { laadher } \\
\text { encia a } \\
\text { la } \\
\text { higiene } \\
\text { de } \\
\text { manos } \\
\text { em un } \\
\text { hospital } \\
\text { universit } \\
\text { ario. }\end{array}$ & $\begin{array}{l}\text { Espanhol/B } \\
\text { VS/2017. }\end{array}$ & $\begin{array}{l}\text { Estudo } \\
\text { transversal } \\
\text { de natureza } \\
\text { descritiva. } \\
\text { Nível de } \\
\text { evidência IV. }\end{array}$ & $\begin{array}{l}\text { O estudo buscou avaliar as intervenções multimodais } \\
\text { que sugerem mudanças estruturais e organizacionais, } \\
\text { treinamento, avaliação dos indicadores de melhoria, } \\
\text { anotações sobre as novas práticas e fortalecimento do } \\
\text { clima de segurança.As intervenções descritas no artigo } \\
\text { estão relacionadas à higienização das mãos e visam } \\
\text { amenizar as taxas de infecções hospitalares. Como } \\
\text { indicadores de melhoria, os pesquisadores analisaram a } \\
\text { taxa de consumo de produto à base de álcool (PBA), } \\
\text { número de dispensadores de PBA próximos aos leitos, } \\
\text { número de práticas relacionadas à higiene das mãos e } \\
\text { número de infecções causadas por Staphylococcus } \\
\text { aureus resistente à meticilina. Os profissionais de saúde } \\
\text { higienizaram as mãos em 54,5\% dos momentos } \\
\text { registrados como oportunos à prática. Concluiu-se } \\
\text { também que após as intervenções multimodais houve } \\
\text { um aumento significativo quanto ao consumo de } \\
\text { produtos à base de álcool e que o número de infecções } \\
\text { diminuiu. }\end{array}$ \\
\hline
\end{tabular}

QUADRO 1- Caracterização dos estudos selecionados. Catalão, GO, Brasil, 2019. Fonte: Elaborado pelos autores. Catalão, GO, Brasil, 2019.

Em 2008, a Organização Mundial da Saúde publicou um manual direcionado às instituições de saúde para a implantação da estratégia multimodal com o intuito de melhorar a prática de higienização das mãos. Essa estratégia multimodal inclui mudanças do sistema, atividades de capacitação, avaliação e feedback do novo sistema, inclusão de lembretes no local de trabalho e consolidação do clima de segurança institucional. Pode-se dizer, então, que a estratégia multimodal trata-se de uma proposta educacional que pode ser aplicada em diferentes contextos com o objetivo de estimular melhorias organizacionais. Contudo, o guia supracitado traz instruções específicas para que os profissionais de saúde tenham boas práticas de higiene (OMS, 2008). Por esse motivo, todos os artigos analisados nesta revisão integrativa referem-se à avaliação dessa estratégia multimodal relacionada à higienização das mãos.

Foram selecionados quatro artigos relacionados à implantação da estratégia multimodal como uma ferramenta para aumentar a prática de higienização das mãos. Todos os estudos partiram do pressuposto de que boas práticas de higiene diminuem as taxas de Infecções Relacionadas à Assistência à Saúde (IRAS) e, portanto, proporcionam resultados positivos voltados à segurança do paciente. $\mathrm{O}$ estudo conduzido por Fariñas-Alvarez e colaboradores (2017) demonstrou que boas práticas de higiene de mãos estão correlacionadas à redução de infecções por Staphylococcus aureus resistente à meticilina.

A segurança do paciente é uma dimensão da qualidade e, devido à competição entre os prestadores de serviços, os usuários estão cada vez mais exigentes e procuram por instituições que zelem pela segurança e bem-estar de seus pacientes. Ademais, as mudanças no cenário mercadológico acontecem de forma rápida e, por isso, as organizações necessitam desenvolver estratégias para acompanhar essas transformações (PADUA-FILHO et al., 2014). Esse novo cenário gerou mudanças na área do saber científico e, como consequência, a forma de realizar a assistência se tornou mais complexa. Contudo, no Brasil, os sistemas de 
saúde ainda precisam aperfeiçoar seus serviços em muitos aspectos (GOMES; MACHADO, 2018).

A temática "segurança do paciente" tornou-se relevante entre pesquisadores de todo o mundo desde a publicação do relatório "To Error is Human: Building a safer health system", em 2000. Conforme mencionado anteriormente, esse relatório indicou que milhares de pessoas morrem todos os anos em consequência de erros na assistência (KOHN et al., 2000). A partir desse contexto, o Programa Nacional de Segurança do Paciente (PNSP) foi criado em abril de 2013 por meio da Portaria MS/GM no 529 e RDC 36, entrando em vigor no mês de julho do referido ano. Essa resolução, desde então, instituiu ações de segurança do paciente em serviços de saúde com o objetivo de reduzir riscos e minimizar os incidentes na área de saúde (BRASIL, 2013).

Um dos objetivos específicos do Programa é "Produzir, sistematizar e difundir conhecimentos sobre segurança do paciente" (BRASIL, 2013). Dessa forma, podese dizer que as atividades de educação ou capacitação contínua favorecem o aumento da qualidade assistencial. Sem dúvidas, o processo de ensino e aprendizagem diário conduz os indivíduos para a percepção e discussão de situações-problema, ampliando, assim, a reformulação do conhecimento voltado às práticas individuais e coletivas. Investir, portanto, na qualificação da equipe multiprofissional resulta em melhoria das práticas assistenciais (SILVA et al., 2016).

Nesse sentido, torna-se fundamental desenvolver métodos como a intervenção multimodal que viabiliza e proporciona com precisão normas e recomendações para a prática clínica, além de permitir a apreciação e discussão dos resultados (BLANCO-MAVILLARD et al., 2018). As organizações de saúde, portanto, devem direcionar esforços para propor estratégias com vistas à promoção de processos educacionais voltados à segurança do paciente, o que proporcionará reflexos de uma cultura de segurança com melhor resultado (SILVA et al., 2016).

Em relação à prática de higienização das mãos, a Organização Mundial da Saúde recomenda que essa ação seja feita em cinco momentos: antes de contatar o paciente, antes da realização de qualquer procedimento, após algum risco de exposição a fluidos biológicos, após contato direto com o paciente e após entrar em contato com áreas próximas ao paciente. Embora a prática de higienização das mãos seja importante para evitar danos aos usuários e a equipe de assistência, muitos estudos demonstram que a taxa de adesão à prática ainda é baixa (OLIVEIRA et al., 2016; ZOTTELE et al., 2017). Os resultados encontrados por Vasconcelos e colaboradores (2018) também indicaram baixa adesão de enfermeiros e técnicos de enfermagem à higienização das mãos e os autores relacionaram esse problema à falta de ações educativas, uma vez que muitos colaboradores não recebiam treinamento há muito tempo.

Outro fator que pode contribuir para a baixa adesão à prática de higienização das mãos é a falta de infraestrutura das unidades. Magnago e colaboradores (2019), por exemplo, apontaram que a falta de pias, dispensadores de sabonete líquido, álcool gel, toalhas e lixeiras dificultam a prática. Por esse motivo, o primeiro passo da estratégia multimodal refere-se à mudança de sistema para que os funcionários envolvidos no cuidado tenham acesso à água corrente, álcool no ponto de assistência, sabonete líquido e papel toalha. A partir desse contexto, pode-se afirmar que a direção deve estar envolvida e não somente a equipe que realiza a assistência ao paciente ou usuário. A gestão de risco, qualidade e segurança do paciente são articuladas entre si (FASSARELLA et al., 2017). 
Os últimos artigos desta revisão descrevem a eficácia de projetos relacionados à implantação da estratégia multimodal em ambientes hospitalares. A pesquisadora Ferreira (2014) avaliou o projeto "Mãos limpas são mãos mais seguras" que consistiu na implantação de três itens da estratégia multimodal em unidades hospitalares do estado de São Paulo. A partir da mudança de sistema, treinamento dos funcionários e utilização de lembretes no local de trabalho, constatou-se que houveram melhorias em vários aspectos relacionados à prática de higienização. O estudo conduzido por Fariñas-Alvarez e colaboradores (2017) também constatou que a estratégia multimodal contribuiu para aumentar a prática de higienização das mãos e minimizar a incidência de infecções.

\section{CONCLUSÃO}

O estudo realizado buscou sintetizar e discutir as principais ações, recomendações e lacunas acerca da estratégia multimodal como proposta educacional e inovadora voltada à segurança do paciente. Concluiu-se que a inclusão de estratégias que promovam a fidelidade às recomendações da Organização Mundial da Saúde através de intervenções multicomponentes e multimodais devem ser encorajadas, com a proposta de implementação de evidências no contexto profissional atraves de indicadores de qualidade.

A principal atribuição exercida por esses profissionais é criar elo entre a equipe da unidade/serviço e o departamento de gestão de risco, qualidade e segurança do paciente. Essa função é imprescindível para garantir a implantação, continuidade e manutenção das iniciativas de segurança do paciente, assim como no monitoramento e divulgação dos resultados associados às iniciativas de melhorias contínuas. É relevante destacar que garantir segurança do paciente é fruto do esforço e comprometimento de toda a equipe envolvida direta ou indiretamente na assistência ao paciente.

Este estudo trouxe informações teóricas sobre estratégia multimodal como proposta educacional e inovadora voltada para segurança do paciente. No entanto, é necessário avançar e obter dados referentes ao campo prático com os profissionais que prestam assistência ao paciente.

\section{REFERÊNCIAS}

ANDRADE, L.E.L.; LOPES, J.M.; SOUZA-FILHO, M.C.M.; VIEIRA-JÚNIOR, R.F.; FARIAS, L.P.C. et al. Cultura de Segurança do Paciente em Três Hospitais Brasileiros com Diferentes Tipos de Gestão. Ciência \& Saúde Coletiva. v. 23, n. 1, p. 161-172, 2018. Disponível em: http://www.scielo.br/scielo.php?script=sci_abstract\&pid=S1413-

81232018000100161\&lng=en\&nrm=iso\&tlng=pt. $\quad$ DOI: $\quad 10.1590 / 1413-$ 81232018231.24392015 .

ANVISA. Agência Nacional de Vigilância Sanitária. Manual para observadores: estratégia multimodal da OMS para a melhoria da higienização das mãos. / Organização Mundial da Saúde; tradução de Sátia Marine - Brasília: Organização Pan Americana da Saúde, 2008.

BLANCO-MAVILLARD,I; VENY, M.B; GOMEZ, J.EP; SUAREZ,A.B.M;GARCIA,G.P et al. Implementation of a Knowledge Mobilization Model to Prevent Peripheral Venous Catheter-related Adverse Events: PREBACP study-a multicenter clusterrandomized trial protocol. Implementation Science. v. 13, n. 1, 2018. Disponível 
em: https://implementationscience.biomedcentral.com/articles/10.1186/s13012-0180792-z DOI 101186/s13012-018-0792-z.

BRASIL, Ministério da Saúde. Portaria no 529, de 1ㅇde abril de 2013. Institui o Programa Nacional de Segurança do Paciente (PNSP). Diário Oficial da União, 2013.

BRASIL, Ministério da Saúde. Resolução RDC n 36, de 25 de julho de 2013. Institui ações para a segurança do paciente em serviços de saúde e dá outras providências. Diário Oficial da União, 2013.

DUARTE, S.C.M.; BESSA, A.T.T.; BÜSCHER, A.; STIPP, M.A.C. Caracterização de erros na assistência de enfermagem em terapia intensiva. Cogitare Enfermagem. $v$. 21, n. 5, p. 1-8, 2016. Disponível em: https://www.researchgate.net/profile/Sabrina_Duarte2/publication/306296458_CARA CTERIZACAO_DE_ERROS_NA_ASSISTENCIA_DE_ENFERMAGEM_EM_TERAPI A_INTENSIVA/links/5be33f9e92851c6b27aedf68/CARACTERIZACAO-DE-ERROSNA-ASSISTENCIA-DE-ENFERMAGEM-EM-TERAPIA-INTENSIVA.pdf. DOI: 10.5380/ce.v21i5.45502.

FARIÑAS-ALVAREZ, C.; PORTAL-MARÍA, T.; FLOR-MORALES,V.; AJAHERRERO, A.; FABO-NAVARRO, M. et al. Estrategia multimodal para laMejora de laAdherencia a la Higiene de Manos em un Hospital Universitario. Revista de Calidad Asistencial, v. 32, n. 1, p. 50-56, 2017. Disponível em: https://www.sciencedirect.com/science/article/pii/S1134282X16301191. DOI: https://doi.org/10.1016/j.cali.2016.06.011.

FASSARELLA, C.S.; FERREIRA, S.S.; CAMERINI, F.G.; HENRIQUE, D.M.; LUNA, A.A. et al. Profissionais Mediadores da Qualidade e Segurança do Paciente como estratégia para o Cuidado Seguro. Revista Mineira de Enfermagem. v. 21, 2017. Disponível em: https://www.researchgate.net/publication/322688597_PROFESSIONAL_PATIENT_Q UALITY_AND_SAFETY_MEDIATORS_AS_A_STRATEGY_FOR_SAFE_HEALTH_C ARE. DŌI: 10.5935/1415-2762.20170078.

FERREIRA, S.A. Implantação do Projeto: Segurança do Paciente "Mãos limpas são mãos mais seguras". Avaliação da etapa 2011-2012. Estado de São Paulo. BEPA. v.11, n. 124, p. 1-18, 2014. Disponível em: https://pesquisa.bvs.br/aps/resource/pt/ses-36366.

GOMES, C. A.; MACHADO, A. G. C. Fatores que influenciam a inovação nos serviços públicos: o caso da Secretaria Municipal de Saúde de Campina Grande. Cadernos Gestão Pública e Cidadania, v. 23, n. 74, 2018. Disponível em: http://bibliotecadigital.fgv.br/ojs/index.php/cgpc/article/view/68005. DOI: http://dx.doi.org/10.12660/cgpc.v23n74.68005.

JHA, A.K.; LARIZGOITIA, I.; AUDERA-LOPEZ, C.; PRASOPA-PLAIZIER, N.; WATERS, $\mathrm{H}$.; et al. The global burden of unsafe medical care: analytic modelling of observational studies. Quality \& Safety. v. 22, p.809-815, 2013. DOI: 10.1136/bmjqs-2012-001748. Disponível em: https://qualitysafety.bmj.com/. 
KOHN, L.T.; CORRIGAN, J.M., DONALDSON, M.C. Committee on Quality of Health Care; Institute of Medicine. To Err is Human: building a safer health system. Washington (DC): National Academy Press; 2000.

LANZILLOTTI, L.S.; DE SETA, M.H.; ANDRADE, C.L.T.; MENDES-JÚNIOR, W.V. Eventos Adversos e Outros Incidentes na Unidade de Terapia Intensiva Neonatal. Ciência \& Saúde Coletiva. v. 20, n. 3, p. 937-946, 2015. Disponível em: http://www.scielo.br/pdf/csc/v20n3/pt_1413-8123-csc-20-03-00937.pdf. 10.1590/1413-81232015203.16912013.

MAGNAGO, T.S.B.S.; ONGARO, J. D.; GRECO, P.B.T.; LANES, T.C.; ZOTTELE, C. et al. Infraestrutura para Higienização das Mãos em um Hospital Universitário. Revista Gaúcha de Enfermagem. v. 40, n. esp, 2019. Disponível em: http://www.scielo.br/scielo.php?script=sci_arttext\&pid=S1983-14472019000200404. DOI: http://dx.doi.org/10.1590/1983-1447.2019.20180193.

MAIA, C.S.; FREITAS, D.R.C.; GALLO, L.G.; ARAUJO, W.N. Notificações de Eventos Adversos Relacionados com a Assistência à Saúde que Levaram a Óbitos no Brasil, 2014-2016. Epidemiologia e Serviços de Saúde. v. 27, n. 2, 2018. Disponível em: http://www.scielo.br/scielo.php?pid=S223796222018000200308\&script=sci_abstract\&tlng=pt. $\quad$ DOI: $\quad$ 10.5123/S167949742018000200004.

MANGILLI, D.C.; ASSUNÇÃO, M.T.; ZANINI, M.T.B.; DAGOSTIN, V.S.; SORATTO, M.T. Atuação ética do enfermeiro frente aos erros de medicação. Enfermagem em Foco. v. 8, n. 1, p. 62-66, 2017. Disponível em: http://revista.cofen.gov.br/index.php/enfermagem/article/view/878. DOI: https://doi.org/10.21675/2357-707X.2017.v8.n1.878.

MENDES, K.D.S.; SILVEIRA, R.C.C.P.; GALVÃO, C.M. Revisão Integrativa: Método de pesquisa para a incorporação de evidências na saúde e na enfermagem. Texto Contexto Enfermagem, v. 17, n. 4, p. 758-764, 2008. Disponível em: http://www.scielo.br/scielo.php?script=sci_arttext\&pid=S0104-07072008000400018. DOI: http://dx.doi.org/10.1590/S0104-07072008000400018.

MELLO, J.F.; BARBOSA, S.F.F. Cultura de Segurança do Paciente em Terapia Intensiva: Recomendações da enfermagem. Texto Contexto Enfermagem. v. 22, n.4, 1124-1133, 2013. Disponível em: http://www.scielo.br/scielo.php?script=sci_arttext\&pid=S0104-07072013000400031. DOI: http://dx.doi.org/10.1590/S0104-07072013000400031.

OLIVEIRA, A.C.; PAULA, A.O.; GAMA, C.S.; OLIVEIRA, J.R.; RODRIGUES, C.D. Adesão à Higienização das Mãos entre Técnicos de Enfermagem em um Hospital Universitário. Revista Enfermagem UERJ. v. 24, n. 2, 2016.Disponível em: http://www.facenf.uerj.br/v24n2/v24n2a15.pdf.

http://dx.doi.org/10.12957/reuerj.2016.9945.

OMS - ORGANIZAÇÃO MUNDIAL DA SAÚDE. Guia para Implementação: Um guia para a implantação da estratégia multimodal da OMS para a melhoria da 
higienização das mãos a observadores: estratégia multimodal da OMS para a melhoria da higienização das mãos. Brasília, DF, 2008.64 p.

PADUA FILHO, W.C.; CARVALHO, C.A.J.; MEIRA, B.M. Inovação como um Desafio para as Empresas de Saúde: A experiência em hospital privado. Revista de Administração Hospitalar e Inovação em Saúde. v. 11, n. 2, p. 58-66, 2014. Disponível em: https://revistas.face.ufmg.br/index.php/rahis/article/view/2008. DOI: https://doi.org/10.21450/rahis.v11i2.2008.

REIS, C.T.; MARTINS, M.; LAGUARDIA, J. A segurança do paciente como dimensão da qualidade do cuidado de saúde: um olhar sobre a literatura. Ciência \& saúde coletiva. v. 18, p. 2029-2036, 2013. Disponível em: https://www.scielosp.org/scielo.php?pid=S1413-

$81232013001500018 \&$ script=sci_arttext\&tIng=en.

SILVA, I.R.; ARANTES, R.B.; DIAS, R.F.S.M.; CARREIRO, M.A. Segurança do Paciente: reflexão teórica sobre a cultura de segurança organizacional. Revista PróUniverSUS. $\quad$ v. $09, \quad$ n. 2, p. 06-10, 2018. Disponível em: http://editora.universidadedevassouras.edu.br/index.php/RPU/article/view/1277.

SILVA, N.D.M.; BARBOSA, A.P.; PADILHA, K.G.; MALIK, A.M. Segurança do paciente na cultura organizacional: percepção das lideranças de instituições hospitalares de diferentes naturezas administrativas. Revista da Escola de Enfermagem da USP, v. 50, n. 3, 2016. Disponível em: http://www.scielo.br/pdf/reeusp/v50n3/pt_0080-6234-reeusp-50-03-0490.pdf. DOI: http://dx.doi.org/10.1590/S0080-623420160000400016.

TOBIAS, G.C.; BEZERRA, A,L.Q.; MOREIRA, I.A.; PARANAGUÁ, T.T.B.; Silva, A.E.B.C. Knowledge of nurses on the culture of patient safety in university hospital. Journal of Nursing UFPE on line. v. 10, n. 3, p. 1071-1079, 2016. https://pdfs.semanticscholar.org/b268/7fd8b985c02444ca6246075bbd762eb4ddd1.p df. DOI: 10.5205/reuol.8702-76273-4-SM.1003201617.

VASCONCELOS, R.O.; ALVES, D.C.I.; FERNANDES, L.M.; OLIVEIRA, J.L.C. Adesão à Higienização das Mãos pela Equipe de Enfermagem em Unidade de Terapia Intensiva. Enfermería Global, n. 50, p. 446-461, 2018. Disponível em: http://scielo.isciii.es/pdf/eg/v17n50/pt_1695-6141-eg-17-50-430.pdf.

WEGNER, W.; SILVA, S.C.; KANTORSKI, K.J.C.; PREDEBON, C.M.; SANCHES, M.O. et al. Educação para Cultura da Segurança do Paciente: Implicações para a formação profissional. Escola Anna Nery.v. 20, n.3, 2016. Disponível em: http://www.scielo.br/scielo.php?pid=S1414-

$81452016000300212 \&$ script=sci abstract\&tlng=pt.

DOI: http://dx.doi.org/10.5935/1414-8145.20160068.

ZOTTELE, C.; MAGNAGO, T.S.B.S.; DULLIUS, A.I.S.; KOLANKIEWICZ, A.C.B.; ONGARO, J.D. Adesão dos Profissionais de Saúde à Higienização das Mãos em Pronto-Socorro. Revista da Escola de Enfermagem da USP. v. 51, 2017. Disponível em: http://www.scielo.br/pdf/reeusp/v51/pt_1980-220X-reeusp-51e03242.pdf. DOI: http://dx.doi.org/10.1590/S1980-220X2016035503242 